\title{
High-speed, high-volume optical communication for aircraft
}

\author{
Christopher Schmidt, Joachim Horwath, Amita Shrestha, \\ Florian Moll, Martin Brechtelsbauer, and Christian Fuchs
}

Transportable ground stations that receive high data volumes from aircraft offer a solution for monitoring unforeseen events.

Free-space optical (FSO) communication links enable near-realtime transmission of high data volumes between aircraft and ground stations. This ultrafast movement of information, which uses high-resolution sensor systems, ${ }^{1}$ has particular applications for disaster management, monitoring natural events, and traffic observation. Usually, data reception is by means of fixed ground stations, but since disasters are not limited to areas where stations are available, we have designed the transportable optical ground station (TOGS), which can be relocated for operation anywhere. $^{1-5}$

Our system includes all components required to set up an optical communication link to an aircraft or satellite. It uses a pneumatically deployable Ritchey-Chrétien-Cassegrain telescope with a main mirror diameter of $60 \mathrm{~cm}$, which is adapted to the needs of long-range aircraft and satellite downlinks. TOGS is equipped with a dual-antenna global positioning system and an inclination sensor to determine its own location, heading, and calibration, and it has supports to enable leveling of the station (see Figure 1). For downlinks, the station uses an optical tracking system, which consists of the telescope itself, a receiving path, and a tracking path with a camera that is controlled by a computer (see Figure 2). ${ }^{2,4}$ In the current setup, the receiver is optimized for data rates of up to $1 \mathrm{Gbps}$.

When the supports are stowed inside the box, TOGS is $2.1 \times$ $1.4 \mathrm{~m}$ in size and weighs approximately $500 \mathrm{~kg}$. We maneuver the system using a special truck, which supports the operation with an air-conditioned control room, additional measurement equipment, and screens. All TOGS's operative components are included in the box, and therefore we can also transport the system in the cargo hold of a civil aircraft.

The transmitting component of the communication system is the Free-space Experimental Laser Terminal II (FELT II), which we installed in our Do228 aircraft (see Figure 3). To achieve

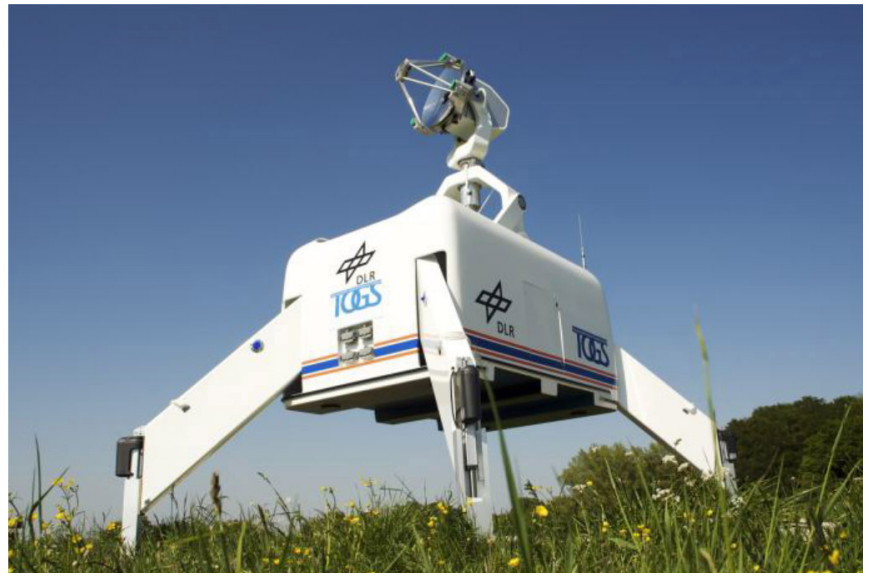

Figure 1. The transportable optical ground station (TOGS) with the telescope unfolded.

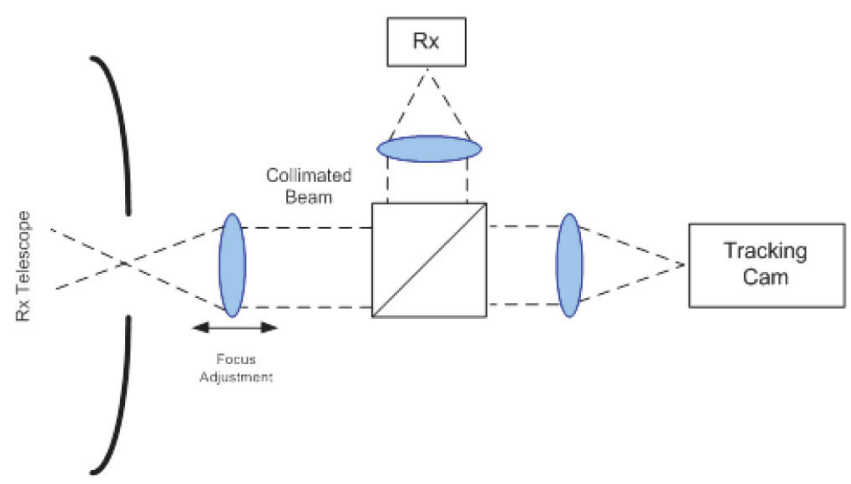

Figure 2. The optical setup of TOGS, which comprises a telescope, a receiving path, and a tracking path. ${ }^{2} \mathrm{R} x$ : Receiver. Cam: Camera.

stable data transmission, the device also requires a tracking system on the transmitter side. The laser terminal consists of a control system with electronic power supply, a computer terminal, microcontrollers (that control the movement of the laser terminal), an inertial measurement unit (for velocity and orientation), the optical bench inside the cabin of the aircraft, and a dome-shaped assembly below the cabin (see Figure 4). FELT II

Continued on next page 


\section{Newsroom}

uses a two-stage tracking system with a fine pointing assembly mounted on the optical bench, and a coarse pointing assembly covered by the dome to protect it from environmental stress during flight.

The transmitting system of the laser terminal is optimized for the data rate of $1 \mathrm{Gbps}$, and has a gigabit Ethernet interface. A field-programmable gate array-based transceiver generates the optical signal. This has a custom-designed transmission protocol capable of overcoming the impairments of FSO communications. ${ }^{5}$

To demonstrate TOGS's capacity in a disaster scenario, we undertook a flight campaign in a rural area near the Bavarian Alps

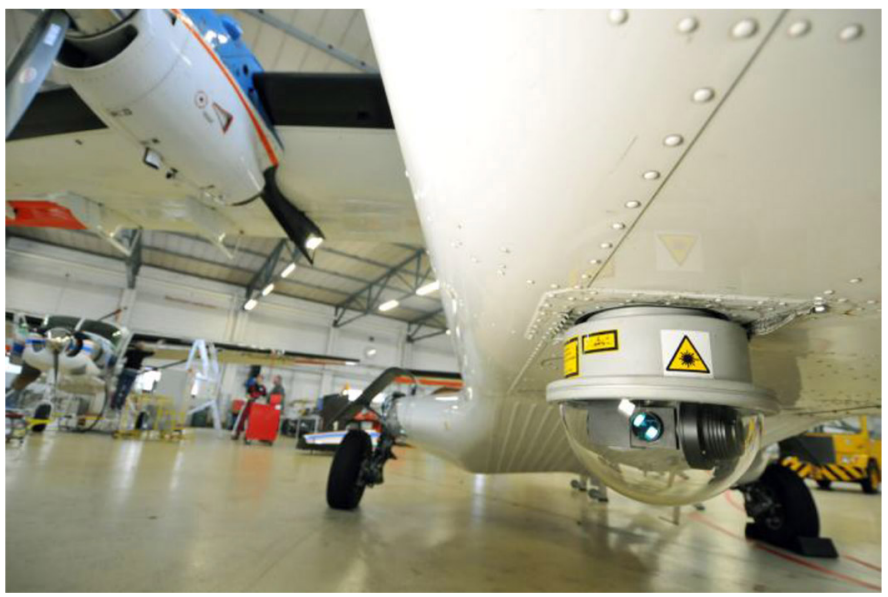

Figure 3. The dome assembly of the Free-space Experimental Laser Terminal II (FELT II) beneath the cabin of the Do228 aircraft.

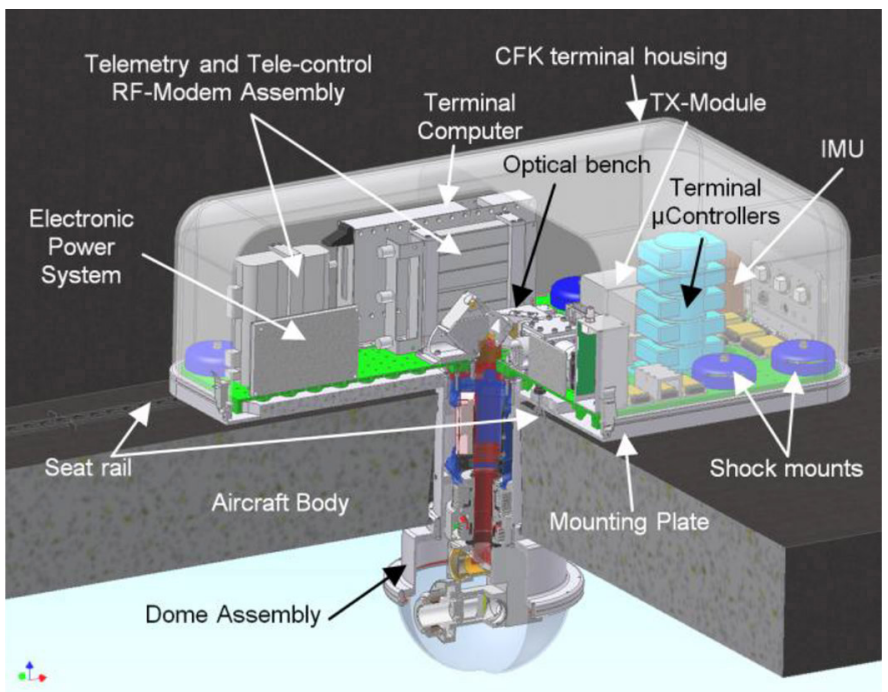

Figure 4. A detailed illustration of FELT II. ${ }^{1}$ RF: Radio frequency. CFK: Carbon-filter-enhanced plastic. TX: Transmission. IMU: Inertial measurement unit.

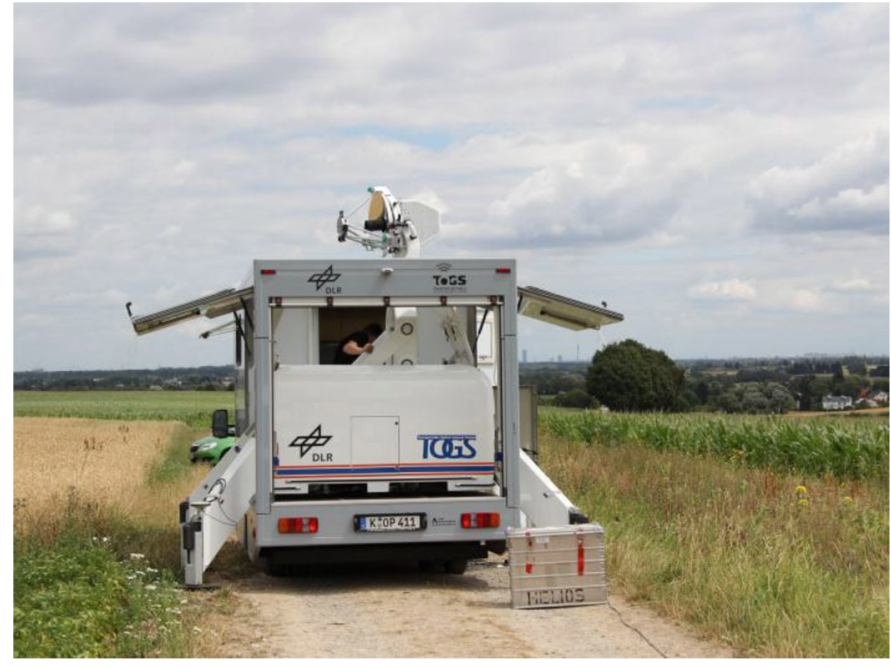

Figure 5. TOGS installed in rural area for data reception from aircraft.

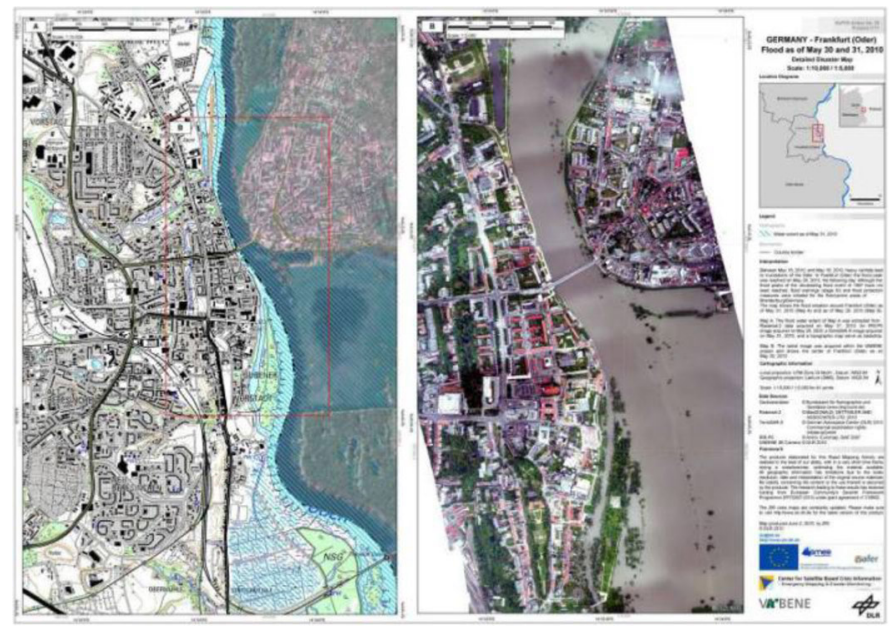

Figure 6. High-resolution images from a flooded area in Germany, generated using TOGS. ${ }^{3}$

(see Figure 5). We set up TOGS to receive data from the Do228 with the installed laser terminal. The data onboard the aircraft comes from a wide-angle camera system, which comprises three cameras arranged to capture a maximum angle of $104^{\circ}$. With an image size of $5616 \times 3744$ pixels and a frame rate of $5 \mathrm{~Hz}$, the optical link transmitted images from the camera onboard to TOGS. ${ }^{3}$ The aircraft flew up to $50 \mathrm{~km}$ with a height of $6500 \mathrm{ft}$ above sea level.

In summary, we have presented a highly portable and adaptable ground station capable of receiving data at a rate of $1 \mathrm{Gbps}$

Continued on next page 
from aircraft, to improve surveillance from the air. The next step for our technology is the development of a smaller and more lightweight laser terminal. This would make the device suitable for use in smaller carriers such as helicopters, enabling greater flexibility for large-scale events and disasters.

The authors would like to thank all members of the German Aerospace Center's flight department, as well as the Optical Communication Systems Group and Advanced Optical Technologies Group at the Institute of Communication and Navigation.

\section{Author Information}

Christopher Schmidt, Joachim Horwath, Amita Shrestha, Florian Moll, Martin Brechtelsbauer, and Christian Fuchs Institute of Communication and Navigation

German Aerospace Center

Oberpfaffenhofen, Germany

Christopher Schmidt holds an MSc in engineering (information and communication technologies) from the University of Erlangen-Nuremberg. He has been a member of the optical communication systems group at the German Aerospace Center since 2011.
References

1. J. Horwath and C. Fuchs, Aircraft to ground unidirectional laser-communications terminal for high resolution sensors, Proc. SPIE 7199, p. 719909, 2009.

doi: $10.1117 / 12.808869$

2. A. Shrestha and M. Brechtelsbauer, Transportable optical ground station for highspeed free-space laser communication, Proc. SPIE 8517, p. 851706, 2012. doi: $10.1117 / 12.928966$

3. F. Kurz et al., Real time camera system for disaster and traffic monitoring, Int'l Conf. Sens. Mod. Photogram. Rem. Sens., 2011.

4. C. Fuchs, M. Brechtelsbauer, J. Horwath, A. Shrestha, F. Moll, D. Giggenbach, and C. Schmidt, DLR's transportable optical ground station, 2013. Paper accepted at Appl. Lasers Sens. Free Space Commun. Conf. in Paris, 27 October-1 November 2013.

5. J. Ramírez, A.Shrestha, S. Parthasarathy, and D. Giggenbach, Gigabit laser ethernet transceiver for free-space optical communication systems, 2013. Paper accepted at Appl. Lasers Sens. Free Space Commun. Conf. in Paris, 27 October-1 November 2013. 